\title{
Simultaneous Isolation and Culture of Atrial Myocytes, Ventricular Myocytes, and Non-Myocytes from an Adult Mouse Heart
}

\author{
Erik A. Blackwood ${ }^{1}$, Alina S. Bilal ${ }^{1}$, Khalid Azizi ${ }^{1}$, Anup Sarakki ${ }^{1}$, Christopher C. Glembotski ${ }^{1}$ \\ ${ }^{1}$ San Diego State University Heart Institute and the Department of Biology, San Diego State University
}

\section{Corresponding Author}

Christopher C. Glembotski

cglembotski@sdsu.edu

\section{Citation}

Blackwood, E.A., Bilal, A.S.,

Azizi, K., Sarakki, A.,

Glembotski, C.C. Simultaneous

Isolation and Culture of Atrial Myocytes,

Ventricular Myocytes, and Non-Myocytes

from an Adult Mouse Heart. J. Vis.

Exp. (160), e61224, doi:10.3791/61224

(2020).

\section{Date Published}

June 14,2020

DOI

$10.3791 / 61224$

URL

jove.com/video/61224

\section{Abstract}

The isolation and culturing of cardiac myocytes from mice has been essential for furthering the understanding of cardiac physiology and pathophysiology. While isolating myocytes from neonatal mouse hearts is relatively straightforward, myocytes from the adult murine heart are preferred. This is because compared to neonatal cells, adult myocytes more accurately recapitulate cell function as it occurs in the adult heart in vivo. However, it is technically difficult to isolate adult mouse cardiac myocytes in the necessary quantities and viability, which contributes to an experimental impasse. Furthermore, published procedures are specific for the isolation of either atrial or ventricular myocytes at the expense of atrial and ventricular non-myocyte cells. Described here is a detailed method for isolating both atrial and ventricular cardiac myocytes, along with atrial and ventricular non-myocytes, simultaneously from a single mouse heart. Also provided are the details for optimal cell-specific culturing methods, which enhance cell viability and function. This protocol aims not only to expedite the process of adult murine cardiac cell isolation, but also to increase the yield and viability of cells for investigations of atrial and ventricular cardiac cells.

\section{Introduction}

Primary cell culture is an integral resource that offers a controlled environment for detailed mechanistic studies of cardiac myocyte function. Due to their more durable nature and ease of isolation, neonatal rat atrial and ventricular myocytes have been the common source of such cell cultures $^{1}$. However, adult mouse atrial and ventricular myocytes (AMAMs and AMVMs) are highly desirable for in vitro studies, because their molecular and functional characteristics better mimic those of adult heart cells. Thus, they have become relevant for studies related to cardiac pathologies, most of which develop in adults ${ }^{2}$.

Furthermore, the availability and use of transgenic and disease mouse models expands the utility of isolated adult cardiac myocytes. Protocols for the isolation and 
culture of mouse AMVMs for short- and long-term studies have been described in numerous previous publications $^{2}, 3,4,5,6,7,8,9,10,11$. In comparison, few protocols have been described for the isolation of AMAMs. Furthermore, those that are described are primarily optimized for acute studies of freshly isolated cells, with no longterm culturing protocol described to date ${ }^{11,12,13}$. As such, AMAM isolation protocols were not designed to provide the utility and versatility of published protocols for the isolation and culture of AMVMs. Furthermore, while the pioneering studies for the isolation of AMAMs and AMVMs have proven resourceful, there are no protocols for optimal concurrent isolation and culture of both AMAMs and AMVMs, which results in efficient use of the whole heart for each preparation.

Until now, published AMAM and AMVM isolation protocols were not designed for simultaneous isolation of both cell types, because most studies on atrial and ventricular function have a chamber-specific focus. For instance, AMAMs are used predominantly to study atrial myocyte electrophysiology, partly because of the interest in atrial fibrillation (AF), the most common cardiac arrhythmia in the U.S. However, AF is not a disease that affects the atria in isolation, and it has been implicated as having a causative role in mild to severe left ventricular dysfunction ${ }^{14}$. Furthermore, electrocardiograms from patients with heart failure with preserved ejection fraction (HFpEF) have illustrated that left atrial size is one of the strongest predictors for susceptibility to heart failure ${ }^{15}$.

In addition to its role in electrophysiology and contractility, the atrium is also an endocrine organ, secreting cardiokines (i.e., atrial natriuretic peptide [ANP]) that homeostatically regulate blood pressure and volume $e^{16,17}$. Moreover, ANP (presumably from atrial myocytes) has a prominent protective and anti-hypertrophic role in ventricular myocytes $^{16,17}$. While there is a strong implication of neurohormonal communication between atria and ventricles in various disease states, the mechanisms underlying this communication has not been fully explored. This point is further exemplified by the surge in research focusing on 1) the role of non-myocytes (specifically cardiac fibroblasts and immune cells) in the diseased heart and 2) how cardiac remodeling as a function of disease directly affects cardiac myocyte viability and global cardiac function $18,19,20,21,22$. Thus, studying cardiac cells from both atria and ventricles is a necessary approach to gain a more complete picture of their roles in cardiac pathophysiology.

The following protocol describes the simultaneous isolation of atrial and ventricular myocytes and non-myocytes from a single mouse heart under physiological and pathophysiological conditions. Additionally, this method is the first to describe optimal conditions necessary for maintaining cultures of atrial cardiac myocytes, as conditions for maintaining cultures of ventricular myocytes have already been published.

\section{Protocol}

All research performed on mice reported in this paper has been reviewed and approved by the SDSU Institutional Animal Care and Use Committee and it conforms to the Guide for the Care and Use of Laboratory Animals published by the National Research Council.

\section{Preparation of isolation and culture media and plating}

1. Prepare $1 \mathrm{~L}$ of heart perfusion media prior to use by adding Joklik modified minimum essential media (MMEM) to $1 \mathrm{~L}$ of sterile water. Adjust the $\mathrm{pH}$ to 7.36 with $10 \mathrm{~N}$ 
$\mathrm{NaOH}$, then filter through a $0.2 \mu \mathrm{m}$ filter and store at $4{ }^{\circ} \mathrm{C}$ for up to 2 weeks.

NOTE: Joklik MMEM consists of $112 \mathrm{mM} \mathrm{NaCl}, 5.4 \mathrm{mM}$ $\mathrm{KCl}, 1 \mathrm{mM} \mathrm{MgCl}_{2}, 9 \mathrm{mM} \mathrm{NaH} \mathrm{PO}_{4}$, and $11.1 \mathrm{mM} \mathrm{D-}$ glucose. This is supplemented with $10 \mathrm{mM}$ HEPES (2.38 g/L), 30 mM taurin (3.75 g/L), 2 mM D-I-carnitine (0.4 g/L), $2 \mathrm{mM}$ creatine, and $10 \mathrm{mM}$ butanedione monoxime (1.01 g/L).

2. Prepare digestion buffer just prior to perfusion, as follows: supplement $50 \mathrm{~mL}$ of heart perfusion media with $6.25 \mu \mathrm{L}$ of $100 \mathrm{mM} \mathrm{CaCl} 2$ (see step 1.3) and collagenase type 2 (enzymatic activity 310-320 U/mg dw).

NOTE: Weigh the animal prior to sacrifice. The amount of collagenase type 2 supplemented into the digestion buffer is dictated by the weight of the animal $(2.25 \mathrm{mg}$ of collagenase type 2 per $1 \mathrm{~g}$ of body weight).

3. To prepare $100 \mathrm{mM} \mathrm{CaCl}_{2}$, add $1.47 \mathrm{~g}_{\text {of }} \mathrm{CaCl}_{2}$ to $100 \mathrm{~mL}$ of molecular biology grade water. Stir until dissolved, then pass through a $0.2 \mu \mathrm{m}$ filter and store at room temperature for up to 2 months.

4. Prepare myocyte stopping buffer 1 by supplementing 90 $\mathrm{mL}$ of heart perfusion buffer with $10 \mathrm{~mL}$ of fetal bovine serum (FBS) and $125 \mu \mathrm{L}$ of $100 \mathrm{mM} \mathrm{CaCl}_{2}$. Pass through a $0.2 \mu \mathrm{m}$ filter and store at $4{ }^{\circ} \mathrm{C}$ for up to 2 weeks.

5. Prepare myocyte stopping buffer 2 by supplementing 114 $\mathrm{mL}$ of heart perfusion buffer with $6 \mathrm{~mL}$ of FBS and $150 \mu \mathrm{L}$ of $10 \mathrm{mM} \mathrm{CaCl} 2$. Pass through a $0.2 \mu \mathrm{m}$ filter and store at $4{ }^{\circ} \mathrm{C}$ for up to 2 weeks.

6. Prepare atrial myocyte plating medium just prior to perfusion by supplementing $95 \mathrm{~mL}$ of Dulbecco's modified Eagle medium (DMEM) with $4 \mathrm{~mL}$ of FBS, $1 \mathrm{~mL}$ of $100 \mathrm{x}$ pen/strep-glutamine, $1 \mathrm{~mL}$ of $100 x$ insulin-transferrin- selenium, and $1 \mathrm{~mL}$ of $10 \mu \mathrm{M}$ dexamethasone. Pass through a $0.2 \mu \mathrm{m}$ filter and store at $37^{\circ} \mathrm{C}$ until plating.

7. Prepare ventricular myocyte plating medium by supplementing $95 \mathrm{~mL}$ of minimum essential medium (MEM) with $4 \mathrm{~mL}$ of FBS, $1 \mathrm{~mL}$ of $100 \mathrm{x}$ pen/strepglutamine, $10 \mathrm{~mL}$ of $1 \mathrm{M}$ HEPES solution, $1 \mathrm{~mL}$ of $100 \mathrm{x}$ insulin-transferrin-selenium, and $0.1 \mathrm{~g}$ of butanedione monoxime. Pass through a $0.2 \mu \mathrm{m}$ filter and store at $4{ }^{\circ} \mathrm{C}$ for up to 2 weeks.

8. Prepare ventricular myocyte maintaining medium by supplementing $99 \mathrm{~mL}$ of MEM with $1 \mathrm{~mL}$ of $100 x$ insulintransferrin-selenium, $0.1 \mathrm{mg} / \mathrm{mL}$ bovine serum albumin (BSA), $10 \mathrm{~mL}$ of $1 \mathrm{M}$ HEPES solution and $1 \mathrm{~mL}$ of $100 \mathrm{x}$ pen/strep-glutamine. Pass through a $0.2 \mu \mathrm{m}$ filter and store at $4{ }^{\circ} \mathrm{C}$ for up to 2 weeks.

9. To prepare laminin-coated experimental plates and slides, thaw mouse laminin stock solution $(1.19 \mathrm{mg} / \mathrm{mL})$. Add $10 \mu \mathrm{L}$ of laminin stock solution to every $1 \mathrm{~mL}$ of DMEM, and mix. Coat experimental plates and slides evenly and store in a $37^{\circ} \mathrm{C}, 5 \% \mathrm{CO}_{2}$ incubator for at least $1 \mathrm{~h}$ prior to perfusion to allow for equilibration.

NOTE: Coated experimental plates and slides can be stored at $4{ }^{\circ} \mathrm{C}$ for up to 2 days.

\section{Isolation apparatus}

1. Prior to each isolation, clean the tubing and other components of the system with three complete washes of $70 \% \mathrm{EtOH}$ by filling the bubble trap to the top (close off the bottom stopcock and keep the top stopcock open).

2. Rinse the complete system $3 x$ with sterile $\mathrm{H}_{2} \mathrm{O}$ by filling the bubble trap to the top (close off the bottom stopcock and keep the top stopcock open). 
3. Rinse out the complete system with heart perfusion buffer and fill the bubble trap halfway with media.

4. Set the circulating water bath to $37^{\circ} \mathrm{C}$.

5. Set a water bath for media to $37^{\circ} \mathrm{C}$.

6. Remove any air bubbles in the peristaltic pump tubing.

7. Adjust the flow rate of the peristaltic pump to $3 \mathrm{~mL} / \mathrm{min}$.

\section{Surgical procedure (non-survival)}

1. Soak surgical instruments in $70 \% \mathrm{EtOH}$ for at least $5 \mathrm{~min}$.

2. Place the heart perfusion media, myocyte stopping buffers, and digestion buffer in the $37^{\circ} \mathrm{C}$ water bath.

3. Place the atrial myocyte plating medium, ventricular myocyte plating medium, and ventricular myocyte maintaining medium in a $37{ }^{\circ} \mathrm{C}, 5 \% \mathrm{CO}_{2}$ incubator $1 \mathrm{~h}$ prior to use and loosen the caps to allow equilibration.

4. Inject 10-week-old male or female C57b6/j mice intraperitoneally (i.p.) with $0.35 \mathrm{~mL}$ of heparin, diluted in phosphate buffered saline (PBS) to $100 \mathrm{IU} / \mathrm{mL}$. Allow the drug to take effect for about $10 \mathrm{~min}$.

NOTE: If two hearts are to be subjected to this isolation procedure, the second mouse can be anesthetized and administered heparin at this point in the first procedure. To minimize stress on the animal, light anesthesia can be administered using $2 \%$ isoflurane/oxygen mixture in a hermetically sealed induction chamber.

5. Anesthetize the animal with a $2 \%$ isoflurane/oxygen mixture and inject i.p. with pentobarbital $(0.3 \mathrm{~mL}$ from 10 $\mathrm{mg} / \mathrm{mL}$ stock), then prepare the chest by swabbing with $70 \% \mathrm{EtOH}$.

6. Prepare a tied $5-0$ silk suture to be ready to ligate the heart to the perfusion cannula. Mount cannula right next to surgical microscope.
7. Quickly open the chest by first making a midline skin incision, from mid-abdomen to the jaw, then entering the peritoneum with the large scissors, clearing the diaphragm away by blunt dissection. Then, cut away the rib cage using the scissors with cuts up the chest wall on the lateral aspect of both sides.

8. Snip away fibrous connections between the heart and chest wall (including thymus). Then, cut away the rib cage all together. Using the small forceps and scissors, gently lift the heart by the apex and expose the posterior aspect of the heart.

9. Explant the heart by dissecting immediately inferior to the innominate artery on the ascending aorta and immediately place the heart in ice-cold PBS or cold heart perfusion media. Subsequently, quickly dissect away remaining tissue from the explanted heart in ice-cold heart perfusion media, exposing the ascending aorta.

NOTE: It is useful to explant the heart with the thymus intact to use as an anatomical landmark.

10. Clean the area surrounding the aorta of excess tissue using micro-dissecting forceps and scissors. Position the aorta onto the cannula using fine-tipped forceps and secure with a 5-0 silk suture.

NOTE: The best placement usually occurs with the aorta extending about $2 \mathrm{~mm}$ up on the cannula.

11. Perfuse the cannulated heart with heart perfusion media at a flow rate of $3 \mathrm{~mL} / \mathrm{min}$ for $4 \mathrm{~min}$. Then switch the media from heart perfusion media to digestion buffer for 15.0-17.5 min of perfusion (Figure 1A).

NOTE: Coronary flow rate will likely increase, indicating effective tissue digestion (i.e., pale, swollen heart).

12. Collect $8 \mathrm{~mL}$ of digestion buffer flow through during the final minutes of perfusion for later use in step 5.4. 
13. Remove the heart from the cannula and place it on a 60 $\mathrm{mm}$ plastic culture dish. Remove excess tissue (i.e., aorta, veins) and submerge the heart in $2.5 \mathrm{~mL}$ of digestion buffer in preparation for mechanical separation.

NOTE: At this point, the atria are dissected away, and one experimenter should conduct the atrial cell isolation protocol, while a second experimenter should conduct the ventricular cell isolation.

\section{Atrial cell isolation and culture}

1. Dissect the atria away from the heart and place it into a $30 \mathrm{~mm}$ plastic culture dish. Submerge it in $0.75 \mathrm{~mL}$ of digestion buffer for mechanical separation. Keep the ventricles in the $60 \mathrm{~mm}$ dish (step 3.12) and carry out the separate isolation methods simultaneously (section 5 , Figure 1B).

NOTE: At this point, the atria and ventricles can undergo further separation should there be a need to isolate the left- and right-side cells.

2. Begin to mince and tease the atria apart, initially with fine-tip surgical scissors and followed by fine forceps for further mincing. Avoid agitating the tissue, and do not rapidly pull apart muscle fibers.

3. Using a sterile transfer pipette tip, continue to gently mix and dissociate the tissue for $15 \mathrm{~min}$. Every $5 \mathrm{~min}$, observe atrial myocyte disassociation from tissue under a 10x objective brightfield microscope. As tissue becomes further digested, continue gently mixing and dissociating tissue using a sterile transfer pipette tip with a smaller pore size.

4. Transfer cell suspension to a $2 \mathrm{~mL}$ sterile micro-centrifuge tube. Rinse the $30 \mathrm{~mm}$ plate with $0.75 \mathrm{~mL}$ of $37{ }^{\circ} \mathrm{C}$ myocyte stopping buffer 1 and combine with the cell suspension (end volume $=1.5 \mathrm{~mL}$ ).

5. Allow the atrial myocytes to sediment by gravity for $10 \mathrm{~min}$ at room temperature. Gently agitate the cell suspension to allow for myocytes to sediment to the bottom of the conical tube, forming a visible pellet.

6. Centrifuge the cell suspension for $5 \mathrm{~min}$ at $20 \times \mathrm{g}$. Carefully remove the supernatant, which contains the non-myocytes, and transfer to a $15 \mathrm{~mL}$ polypropylene conical tube using a sterile pipette tip without disturbing the pellet of atrial myocytes.

7. Centrifuge the non-myocyte fraction for $5 \mathrm{~min}$ at 20,000 $x g$. Aspirate the supernatant and resuspend the nonmyocyte pellet in $10 \mathrm{~mL}$ of DMEM supplemented with $10 \%$ fetal calf serum (FCS).

8. Count non-myocytes using a hemocytometer or other method, then plate as per experimental needs, or further isolate into individual specific cell populations via fluorescence-activated cell sorting (Figure 1C).

9. Resuspend the pellet of isolated atrial myocytes from step 4.6 in $1 \mathrm{~mL}$ of atrial myocyte plating medium and apply 10 $\mu \mathrm{L}$ of this suspension onto a hemocytometer. Perform a cell count of rod-shaped myocytes per field.

10. Aspirate laminin from precoated experimental plates/ slides and resuspend the isolated atrial myocytes in the appropriate volume of atrial myocyte plating medium supplemented with $25 \mu \mathrm{M}$ blebbistatin. Plate at the desired density per experimental needs (Figure 1C).

NOTE: Typical plating density for the long-term culturing described here is $5 \times 10^{5}$ cells/chamber on four-chamber $\left(1.7 \mathrm{~cm}^{2}\right)$ glass slides. 


\section{Ventricular cell isolation and culture}

1. Begin to mince and tease heart apart ventricular tissue, first with fine-tip surgical scissors and followed by fine forceps for further mincing (Figure 1B). Avoid agitating the tissue by rapidly pulling apart muscle fibers.

2. Transfer the cell suspension to a $15 \mathrm{~mL}$ polypropylene conical tube. Rinse the plate with $2.5 \mathrm{~mL}$ of $37^{\circ} \mathrm{C}$ myocyte stopping buffer 1 and combine with the cell suspension (end volume $=5 \mathrm{~mL}$ ).

3. Using a sterile transfer pipette tip, continue to gently mix and dissociate the tissue for $4 \mathrm{~min}$. Apply $10 \mu \mathrm{L}$ of this cell suspension onto the slide and visualize the presence of rod-shaped myocytes to ensure the quality of isolation.

4. Pass the cell suspension through a $100 \mu \mathrm{m}$ sterile nylon filter into a $50 \mathrm{~mL}$ polypropylene conical tube. Use $2 \mathrm{~mL}$ of digestion buffer collected in step 3.12 to wash any remaining cells off the sterile nylon filter.

5. Allow the ventricular myocytes to sediment by gravity for $6 \mathrm{~min}$ at room temperature. Gently agitate the filtered cell suspension to allow for myocytes to sediment at the bottom of the conical, forming a visible pellet.

6. Without disturbing the pellet of ventricular myocytes, carefully remove the supernatant (non-myocytes) and transfer to a $50 \mathrm{~mL}$ polypropylene conical tube using a sterile pipette tip. Centrifuge the non-myocyte fraction for $5 \mathrm{~min}$ at $20,000 \times \mathrm{g}$. Aspirate the supernatant and resuspend the non-myocyte pellet in $10 \mathrm{~mL}$ of DMEM supplemented with $10 \%$ FCS.

7. Count non-myocytes using a hemocytometer and resuspend in an appropriate volume of DMEM supplemented with $10 \%$ FCS. Then, plate according to experimental needs, or further isolate into individual specific cell populations via fluorescence-activated cell sorting (Figure 1C).

8. Resuspend the isolated ventricular myocytes in $2 \mathrm{~mL}$ of myocyte stopping buffer 2 and apply $10 \mu \mathrm{L}$ of cell suspension onto hemocytometer. Perform a cell count of rod-shaped myocytes per field.

9. Reintroduction of $\mathrm{Ca}^{2+}$ using a stepwise paradigm NOTE: Calcium reintroduction steps are specific for ventricular myocytes and should not be performed for atrial myocytes, as this will result in cell death.

1. Add $50 \mu \mathrm{L}$ of $10 \mathrm{mM} \mathrm{CaCl}_{2}$ to the ventricular myocyte cell suspension. Mix well and incubate for 4 min at room temperature.

2. Add an additional $50 \mu \mathrm{L}$ of $10 \mathrm{mM} \mathrm{CaCl}_{2}$ to the ventricular myocyte cell suspension. Mix well and incubate for $4 \mathrm{~min}$ at room temperature.

3. Add an additional $100 \mu \mathrm{L}$ of $10 \mathrm{mM} \mathrm{CaCl}_{2}$ to the ventricular myocyte cell suspension. Mix well and incubate for $4 \mathrm{~min}$ at room temperature.

4. Add $80 \mu \mathrm{L}$ of $100 \mathrm{mM} \mathrm{CaCl} 2$ to the ventricular myocyte cell suspension. Mix well and incubate for 4 min at room temperature.

10. Remove laminin coating from plates or slides and resuspend the isolated ventricular myocytes in an appropriate volume of ventricular myocyte plating medium according to experimental needs (Figure 1C).

NOTE: Typical plating density for the long-term culturing described here is $5 \times 10^{5}$ cells/chamber on fourchamber $\left(1.7 \mathrm{~cm}^{2}\right)$ glass slides. Avoid plating at a density of greater than $75 \%$ confluency, as cellular overdensification promotes cell clumping, thereby inhibiting attachment to the plate. Furthermore, large numbers 
of cells will be lost during the first medium change. Plate cells quickly after isolation, as extracellular $\mathrm{Ca}^{2+}$ promotes hypercontraction and loss of viable myocytes.

11. Allow the ventricular myocytes to settle and adhere for at least $1 \mathrm{~h}$. Subsequently change the media to ventricular myocyte maintaining medium supplemented with $25 \mu \mathrm{M}$ blebbistatin.

NOTE: Ventricular myocytes can be cultured up to $96 \mathrm{~h}$ after plating in $\mu \mathrm{M}$ blebbistatin. Experiments should be conducted in ventricular myocyte maintaining medium in the absence of blebbistatin.

\section{Representative Results}

A wildtype 10-week-old C57b6/j mouse heart typically results in between 75,000-150,000 atrial myocytes and 1.0-1.5 x $10^{6}$ ventricular myocytes, equating to an approximate yield of $30 \%-50 \%$ for atrial and ventricular myocytes ${ }^{18}, 19$. During and immediately after isolations, viable cardiac myocytes should appear rod-shaped and non-contracting. A majority of isolated cardiac myocytes should adapt this morphology, which is an indication of effective perfusion. The rodshape morphology can also be a predictor of viability. The protocol aims to enhance the yield and viability of myocytes and non-myocytes isolated from a diseased mouse heart. Furthermore, it has been tested in a model of pressure overload-induced heart failure (data not shown).

To confirm adequate and replicable isolation of myocytes and non-myocytes from atrial and ventricular tissue, cells were observed and photographed at various days in culture (Figure 2). Additionally, quantitative reverse-transcription polymerase chain reaction ( $q R T-P C R)$ was performed to measure the levels of transcripts that were cell typespecific. Cardiac muscle troponin $\mathrm{T}($ Tnnt2) is a marker of cardiac myocytes and was robustly expressed in both atrial and ventricular cardiac myocyte cultures (Figure 3A). In contrast, atrial natriuretic peptide (Nppa, which is typically expressed exclusively in adult atrial cardiac myocytes under physiological conditions) and myosin light chain 2 (Myl2, which is a ventricular myocyte specific gene) were robustly and specifically expressed in atrial and ventricular cardiac myocyte cultures, respectively (Figure 3B,C).

Fibroblast markers, transcription factor 21 (Tcf21), plateletderived growth factor receptor A (Pdgfra), and monocytederived cell marker cluster of differentiation 68 (Cd68) were exclusively expressed in non-myocyte cultures isolated from both atrial and ventricular chambers (Figure 3D-F). It is estimated that non-myocytes compromise $\sim 65 \%$ of all heart cells and that a majority of these originate from a fibroblast or monocyte-derived lineage $18,19,23,24$. Thus, markers for these two lineages were chosen to be representative, given the interest in these cellular populations in studies of various models and etiologies of cardiac pathology.

Immunostaining of AMAMs and AMVMs for the t-tubule marker dihydropyridine (DHPR, which is a voltage-dependent (L)-type calcium channel) as well as the ryanodine receptor (RYR2) demonstrated intact t-tubules throughout isolation and long-term culture (Figure 4A,B). The abundance of DHPR and localization that was characteristic and unique to atrial and ventricular myocytes indicated the presences of t-tubules. Moreover, colocalization of DHPR with RYR2 immunostaining was an indicator of intact diad structures. Immunostaining for the sarcomeric protein alpha-actinin in atrial and ventricular cardiac myocytes resulted in the expected sarcomeric striation pattern. The sarcomeric striation pattern was used to assess the purity and viability of isolated cardiac myocytes in conjunction with rod-shaped morphological shape and nuclear staining with TOPRO-3 
(Figure 4C,D; purple and red). As expected, ventricular cardiac myocytes were large, exhibiting an average length of $\sim 150 \mathrm{~mm}$, whereas atrial cardiac myocytes averaged $\sim 75 \mathrm{~mm}$. Furthermore, upon immunostaining analysis, atrial cardiac myocytes (but not ventricular cardiac myocytes) exhibited robust expression of atrial natriuretic peptide (ANP) in a staining pattern that was characteristic of localization to the endoplasmic reticulum and secretory granules (Figure 4C,D; green).

A characteristic unique to atrial cardiac myocytes is its classification as an endocrine cell in addition to contractile cell. While atrial myocytes secrete ANP under basal conditions, secretion increases in response to secretagogues (i.e., the alpha-adrenergic agonist, phenylephrine [PE]). Moreover, atrial cardiac myocytes secrete ANP and cosecretionally process a portion of the hormone from its precursor state (Pro-ANP, $15 \mathrm{kD}$ ) to the product peptide $(\text { ANP } 3 k D)^{16,17}$. This secretory ability can be quantified via immunoblot detection of ANP in the media of isolated atrial cardiac myocytes in response to acute PE treatment (Figure 4E). This secretory and processing ability of the atrial cardiac myocyte was found to be sensitive to culturing conditions. Thus, it is imperative that the atrial myocyte plating medium is supplemented with dexamethasone, insulin, transferrin, and selenium. 
A. Heart Perfusion

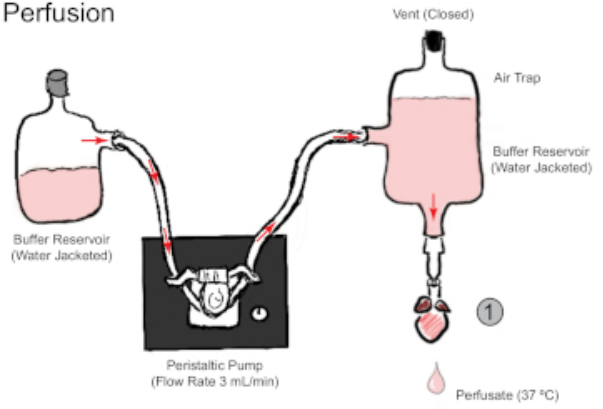

B. Tissue Digestion

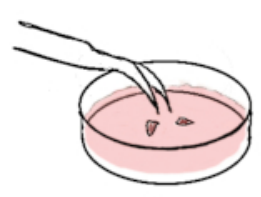

Atrial Tissue

C. Cell Isolation

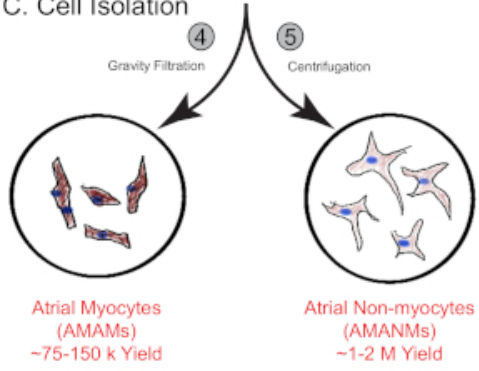

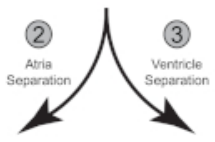

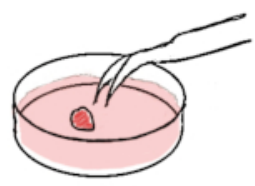

Ventricular Tissue

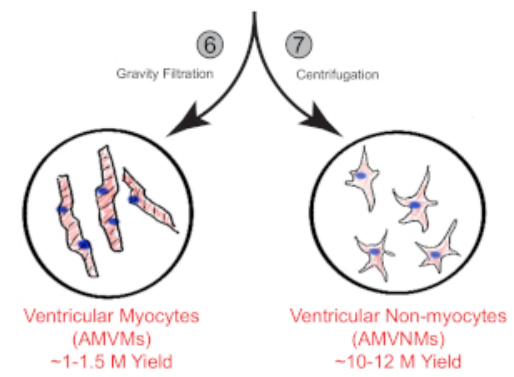

Figure 1: Schematic overview of retrograde heart perfusion, digestion, and cell isolation. Shown are the main steps involved in cell isolation from both atrial and ventricular chambers simultaneously from a single mouse heart. (A) A single mouse heart is rapidly cannulated via the ascending aorta and perfused in a retrograde manner. (B) The heart is separated into atrial and ventricular tissues for further digestion and physical separation. (C) Following adequate digestion, the cells are separated via gravity filtration into a total of four cellular fractions that are cultured for subsequent experimentation. Please click here to view a larger version of this figure. 


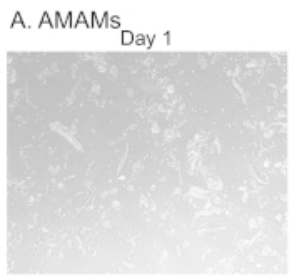

B. AMANMs

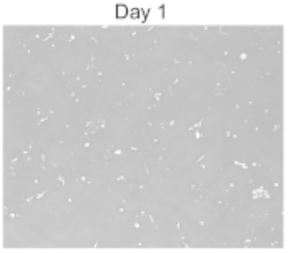

C. AMVMs

Day 1

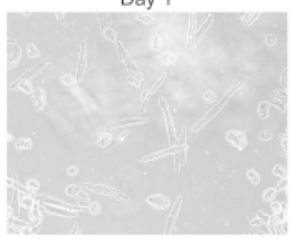

D. AMVNMs

Day 1

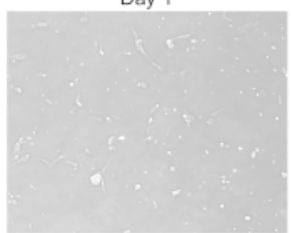

Day 2

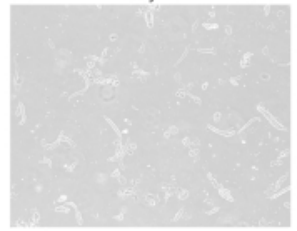

Day 2

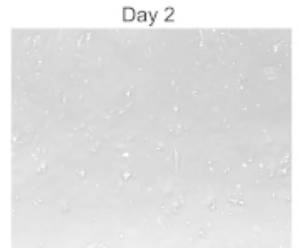

Day 2

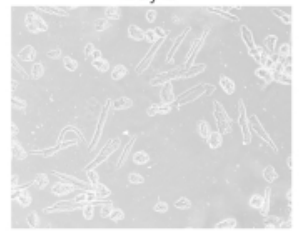

Day 2

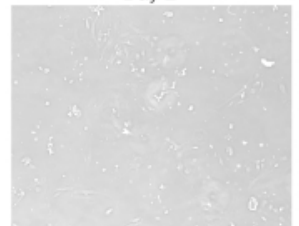

Day 3

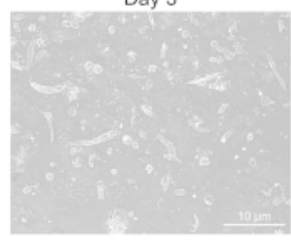

Day 4

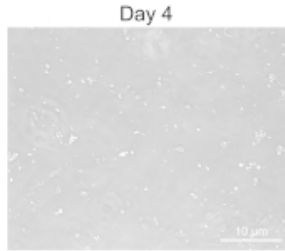

Day 3

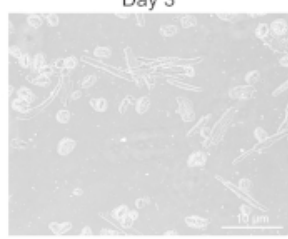

Day 4

Figure 2: Morphological analysis of isolated atrial and ventricular cardiac myocytes and non-myocytes in culture.

(A) Isolated adult mouse atrial myocytes (AMAMs), (B) adult mouse atrial non-myocytes (AMANMs), (C) adult mouse ventricular myocytes (AMVMs), or (D) adult mouse ventricular non-myocytes (AMVNMs) were plated at $5 \times 10^{5}$ cells/ chamber on four-chamber $\left(1.7 \mathrm{~cm}^{2}\right)$ glass slides in respective plated media. Phase images were obtained at indicated days in culture using a 10x objective under an epifluorescence microscope. Please click here to view a larger version of this figure. 
A.

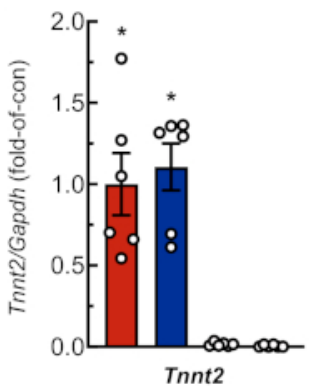

D.

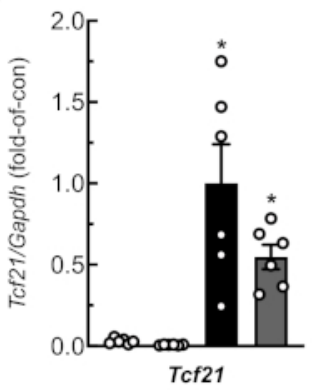

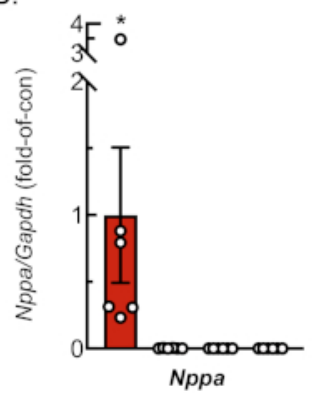

E.

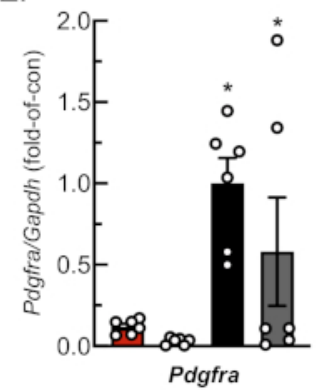

C.

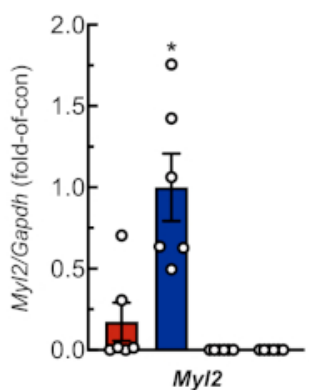

F.

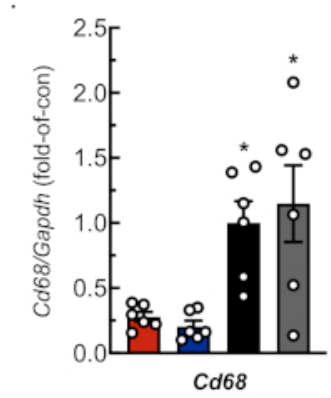

Figure 3: Representative qRT-PCR analysis of isolated cell cultures. RNA was extracted from freshly isolated cardiac myocytes and non-myocytes, and mRNA levels for cell-specific gene markers were determined by qRT-PCR ${ }^{4}$. (A) Tnnt2, cardiac muscle troponin T (cardiac myocyte marker); (B) Nppa, atrial natriuretic peptide (atrial myocyte marker); (C) Myl2, myosin light chain 2 (ventricular myocyte marker); (D) Tcf21, transcription factor 21 (fibroblast marker); (E) Pdgfra, plateletderived growth factor receptor A (fibroblast marker); (F) Cd68, cluster of differentiation 68 (monocyte-derived cell marker). Data represent mean \pm SEM ( ${ }^{*} p \leq 0.05$ different from all other values, as determined by ANOVA followed by Newman Keul's post-hoc analysis). Please click here to view a larger version of this figure. 
A. AMAMs

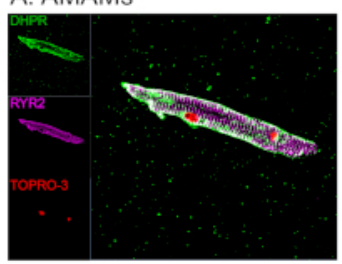

C. AMAMs

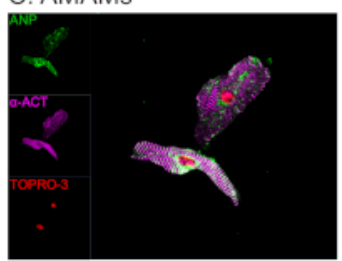

D. AMVMs

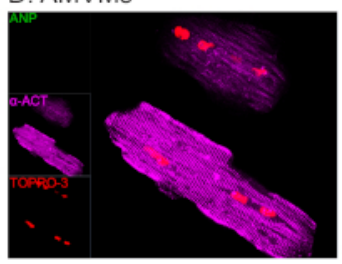

E. AMAM Media

\section{B. AMVMs}
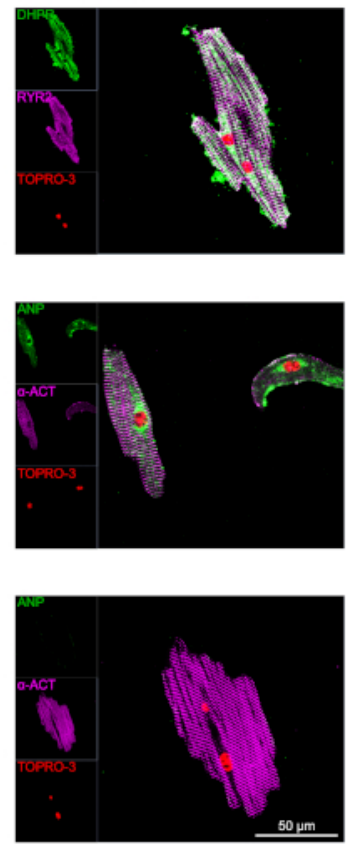

Vehicle $\mathrm{PE}(0.5 \mathrm{~h}) \mathrm{kD}$

Pro-ANP

ANP

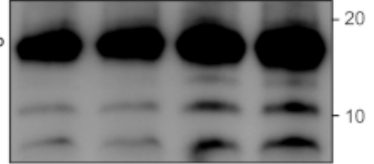

Figure 4: Representative morphological and functional analysis of isolated atrial and ventricular cardiac myoyctes. (A) AMAMs or (B) AMVMs were plated at $5 \times 10^{5}$ cells/chamber on four-chamber $\left(1.7 \mathrm{~cm}^{2}\right)$ glass slides in respective plating media for $1 \mathrm{~h}$ to allow for adhesion. This was followed by either refeeding atrial myocyte plating media or changing to ventricular myocyte maintaining media supplemented with blebbistatin for an additional $16 \mathrm{~h}$. Cultures were subsequently fixed then immunostained for RYR2 (purple), DHPR (green), and nuclear stain TOPRO-3 (red). (C) AMAMs or (D) AMVMs were isolated and plated, then immunostained for a-actinin (purple), ANP (green), and TOPRO-3 (red). Shown are two representative images for each cell type. (E) AMAMs were plated at $5 \times 10^{5}$ cells/well on a 12 well culture dish for 16 $\mathrm{h}$ in atrial myocyte plating media. AMAMs were subsequently treated for $0.5 \mathrm{~h}$ with vehicle or the ANP secretagogue (phenylephrine, $50 \mathrm{mM}$ ) before media were collected and subjected to immunoblot analysis for ANP. Prior to immunoblot analysis, media samples were centrifuged at $500 \times \mathrm{g}$ for $5 \mathrm{~min}$ to remove cellular debris and ensure that the observed ANP was the result of active secretion from AMAMs. Please click here to view a larger version of this figure. 


\section{Discussion}

The quality of the cells isolated using the procedure described here, as determined by the cell yield and overall health of the cells in culture, depends on numerous controllable factors. Starting with the mouse itself, it has been documented that stress imposed on the animal can negatively affect cell yield and viability in culture, presumably due to excess systemic cortisol, catecholamines, and the hypercontractile state of cardiac tissue $^{2,5,7}$. For these reasons, measures should be taken to avoid alarming the animal prior to sacrifice. Such measures include covering the animal's cage and limiting time outside of vivarium prior to sacrifice. Heparin and many barbiturates commonly used for euthanasia can affect signaling pathways; thus, the optimal method of euthanasia should be customized accordingly. The age of the animal has a considerable impact on the quality and viability of isolated cells, most likely due to progressive accumulation of interstitial fibrosis occurring concurrently with the aging process, which can affect tissue digestion ${ }^{25}$. In data not presented here, while the method described above works in mice of up to 78 weeks old, the quality of the cells was lower in these older animals.

The most critical step in the isolation process described, as well as other protocols featuring a Langendorff apparatus for retrograde perfusion, is the cannulation and initial perfusion of the heart. For optimum results, the time from cardiac explantation to cannulation of the ascending aorta and initiation of perfusion should take no more than $90 \mathrm{~s}$. In addition to time, two additional important factors are the depth of cannula and possibility of introducing air emboli from the perfusion apparatus. Accordingly, the cannula should be advanced into the ascending aorta so as not to enter the aortic root and obstruct the aortic valve, which would impair perfusion of the coronary vessels.

During the digestion process, it is important to regularly test the rigidity of the heart to avoid prolonged exposure to the digestive enzyme collagenase, which reduces cardiac myocyte calcium tolerance. The protocol described above for calcium reintroduction into the isolated ventricular myocyte cultures was designed to limit cardiac myocyte death via inappropriate calcium influx via store operated calcium channels. It should be noted that the stepwise calcium reintroduction should not be performed for isolated atrial myocyte cultures, as this will promote cell death during shortand long-term culture ${ }^{12}$. For further precaution, the perfusion and digestion buffers used here include the cardiac muscle contraction inhibitor butanedione monoxime (BDM) to avoid hypercontraction of isolated myocytes, as well as the calcium paradox, both of which impact myocyte viability ${ }^{26}$. However, the switch from BDM to blebbistatin should be noted, as it is the preferred anti-contractile agent in maintaining media for isolated cardiac myocytes. In data not shown, blebbistatin confers greater viability for long-term culture of isolated cardiac myocytes.

Immediately after isolation, it is important to consider the ramifications of long-term culture of cardiac cells, especially myocytes. The cardiac non-myocyte isolation and culturing protocol described here is based on common methods that take advantage of the different densities and adhesive properties of different cardiac cells. The benefit of nonmyocytes is their high expansion potential in culture; thus, unlike cardiac myocytes, they are amenable to passaging for perpetuation. However, it is known that culturing conditions, including medium supplementation with FBS, can affect cardiac myocyte functionality ${ }^{27}$. The culture media described 
here were designed to optimize viability and limit functional derangements, especially for the isolated atrial myocytes. While no overt impaired contractile ability was observed in isolated cardiac myocytes after culture in the absence of blebbistatin supplementation, studies that focus on electrophysiology, contractility, and other single-cell in vivobased molecular signaling should be performed soon after isolation, when the sarcomeric structure and molecular signature still mimics that of the intact heart.

A hallmark feature of the atrial myocyte is its ability to moonlight as an endocrine cell with immense secretory capacity, in addition to their contractile function. Under physiological conditions, atrial myocytes produce large quantities of ANP, which is stored in the endoplasmic reticulum and in large dense-core secretory granules poised for regulated exocytosis upon receiving a stimulus ${ }^{16,17}$. While many isolated atrial myocyte studies focus on their unique electrophysiological properties, this is the first study to design a culture media. This allows for long-term viability as well as promotion of the maintained functions of endocrine and contractile properties of atrial myocytes. This novel method for culturing, as well as simultaneous isolation of all cell types from atrial and ventricular chambers from a single mouse heart, will be useful and efficacious for studies on the physiological and pathophysiological properties of both atrial and ventricular myocytes.

\section{Disclosures}

The authors have nothing to disclose.

\section{Acknowledgments}

E.A.B. was supported by the National Institutes of Health (1F31HL140850), the ARCS Foundation, Inc., San Diego Chapter, and is a Rees-Stealy Research Foundation Phillips
Gausewitz, M.D. Scholar of the SDSU Heart Institute. E.A.B. and A.S.B. were supported by the Inamori Foundation. CCG by (NIH) grants R01 HL135893, R01 HL141463 and HL149931.

\section{References}

1. Peter, A. K., Bjerke, M. A., Leinwand, L. A. Biology of the cardiac myocyte in heart disease. Molecular Biology of the Cell. 27 (14), 2149-2160 (2016).

2. Kruppenbacher, J. P., May, T., Eggers, H. J., Piper, H. M. Cardiomyocytes of adult mice in long-term culture. Naturwissenschaften. 80 (3), 132-134 (1993).

3. Ackers-Johnson, M. et al. A Simplified, LangendorffFree Method for Concomitant Isolation of Viable Cardiac Myocytes and Nonmyocytes From the Adult Mouse Heart. Circulation Research. 119 (8), 909-920 (2016).

4. Jin, J. K. et al. ATF6 decreases myocardial ischemia/ reperfusion damage and links ER stress and oxidative stress signaling pathways in the heart. Circulation Research. 120 (5), 862-875 (2017).

5. Judd, J., Lovas, J., Huang, G. N. Isolation, Culture and Transduction of Adult Mouse Cardiomyocytes. Journal of Visualized Experiments. (114), e54012 (2016).

6. Li, D., Wu, J., Bai, Y., Zhao, X., Liu, L. Isolation and Culture of Adult Mouse Cardiomyocytes for Cell Signaling and in vitro Cardiac Hypertrophy. Journal of Visualized Experiments. (87), e51357 (2014).

7. O'Connell, T. D., Ni, Y. G., Lin, K. M., Han, H. P., Yan, Z. Isolation and culture of adult cardiac myocytes for signaling studies. AfCS Research Reports. 1 (5), 1-9 (2003). 
8. Pinz, I., Zhu, M., Mende, U., Ingwall, J. S. An improvised isolation procedure for adult mouse cardiomyocytes. Cell Biochemistry and Biophysics. 61 (1), 93-101 (2011).

9. Piper, H. Culturing of calcium stable adult cardiac myocytes. Journal of Molecular and Cellular Cardiology. 14 (7), 397-412 (1982).

10. Shioya, T. A simple technique for isolating healthy heart cells from mouse models. Journal of Physiological Sciences. 57 (6), 327-335 (2007).

11. Omatsu-Kanbe, M., Yoshioka, K., Fukunaga, R., Sagawa, H., Matsuura, H. A simple antegrade perfusion method for isolating viable single cardiomyocytes from neonatal and aged mice. Physiological Reports. 6 (9), e13688 (2018).

12. Jansen, H. J., Rose, R. A. Isolation of Atrial Myocytes from Adult Mice. Journal of Visualized Experiments. (149), e59588 (2019).

13. Yao, C. et al. Enhanced Cardiomyocyte NLRP3 Inflammasome Signaling Promotes Atrial Fibrillation. Circulation. 138 (20), 2227-2242 (2018).

14. Cha, Y., Redfield, M. M., Shen, W., Gersh, B. J. Atrial Fibrillation and Ventricular Dysfunction: A Vicious Electromechanical Cycle. Circulation. 109 (23), 2839-2843 (2004).

15. Issa, O. et al. Left atrial size and heart failure hospitalization in patients with diastolic dysfunction and preserved ejection fraction. Journal of Cardiovascular Echography. 27 (1), 1-6 (2017).

16. de Bold, A.J. Atrial natriuretic factor: a hormone produced by the heart. Science. 230 (4727), 767-770 (1985).

17. McGrath, M. F., de Bold, M. L., de Bold, A. J. The endocrine function of the heart. Trends in Endocrinology and Metabolism. 16 (10), 459-477 (2005).
18. Doevendans, P. A., Daemen, M. J., de Muinck, E. D., Smits, J. F. Cardiovascular phenotyping in mice. Cardiovascular Research. 39 (1), 34-49 (1998).

19. Banerjee, I., Fuseler, J. W., Price, R. L., Borg, T. K., Baudino, T. A. Determination of cell types and numbers during cardiac development in the neonatal and adult rat and mouse. American Journal of Physiology. 293, H1883-1891 (2007).

20. Jugdutt, B. I. Ventricular remodeling after infarction and the extracellular collagen matrix: when is enough enough? Circulation. 108, 1395-1403 (2003).

21. Omatsu-Kanbe, M. et al. Identification of cardiac progenitors that survive in the ischemic human heart after ventricular myocyte death. Scientific Reports. 7, 41318 (2017).

22. Song, K. et al. Heart repair by reprogramming nonmyocytes with cardiac transcription factors. Nature. $\mathbf{4 8 5}$, 599-604 (2012).

23. Limana, F. et al. bcl-2 overexpression promotes myocyte proliferation. Proceedings of the National Academy of Sciences of the United States of America. 99, 6257-6262 (2002).

24. Pinto, A. R. et al. Revisiting cardiac cellular composition. Circulation Research. 118 (3), 400-409 (2015).

25. Chen, W., Frangogiannis, N. G. The role of inflammatory and fibrogenic pathways in heart failure associated with aging. Heart Failure Reviews. 15, 415-422 (2010).

26. Daly, M. J., Elz, J. S., Nayler, W. G. Contracture and the calcium paradox in the rat heart. Circulation Research. 61, (4), 560-569 (1987).

27. Li, Z., Sharma, R. V., Duan, D., Davisson, R. L. Adenovirus-mediated gene transfer to adult mouse 
cardiomyocytes is selectively influenced by culture medium. Journal of Gene Medicine. 5 (9), 765-772 (2003). 